\title{
Schizophrenia in Older Adults
}

Elizabeth Collier, BSc, MSc, RMN PGCE

Lecturer in Mental Health

School of Nursing, Midwifery and Social Work

University of Salford

Salford, Greater Manchester United Kingdom

Jeanne M. Sorrell, PhD, RN, FAAN

Senior Nurse Researcher

Department of Nursing Research and Innovation

Cleveland Clinic, Cleveland, Ohio 


\begin{abstract}
Although the number of persons over the age of 55 with schizophrenia is expected to double over the next 20 years, the research data on older people with schizophrenia is limited. This appears to be because until the middle of the $20^{\text {th }}$ century, it was assumed that mental illness in older people was a part of the aging process and older people are often excluded from research investigations. There is a need for nursing research to explore how people with schizophrenia, as they age, learn to manage their problems, as well as how those who are first diagnosed with schizophrenia in later life adapt to their illness. Mental health nurses need to be cautious in assigning premature labels to older adults with mental illness that may lead to unsubstantiated assumptions about levels of disability. Instead, they should realize individual potential regarding undiscovered strengths and should attempt to create interventions that recognize and foster personal development for older adults with schizophrenia.
\end{abstract}




\section{Schizophrenia in Older Adults}

\section{Introduction}

The number of persons over the age of 55 with schizophrenia is expected to double over the next 20 years, from 500,000 to 1 million (Cohen, Vahia, Reyes, Diwan, Bankole, Palekar, Kehn, \& Ramirez, 2008). There is limited research data on older people with schizophrenia, however, continuing a 'benign neglect' highlighted by Bridge, Cannon, and Wyatt in 1978. Research prior to 1987 excluded older people because of diagnostic restrictions and beliefs that a diagnosis of schizophrenia meant onset before the age of 45 (Palmer, Heaton, \& Jeste, 1999). The exclusion of older people with schizophrenia from research developments largely continued until the emergence of a more systematic effort to study this population in the 1990s (Harvey, 2005; Howard, Rabins, Seeman, \& Jeste, 2000).

Schizophrenia, a disease marked by delusions, hallucinations, and disordered thinking, is a functional mental illness, along with bipolar disorder, anxiety disorders, and clinical depression. Functional illnesses are distinct from dementing illnesses (organic), such as Alzheimer's disease, Huntington's chorea, and Lewy body dementia. Research on functional mental illness in older people accounts for only small proportions of published work on later life mental illness, compared to that on younger people or dementia (Collier, 2005). However, it is now recognized that the development of a functional mental illness in later life for the first time is distinct from those diagnosed early in life and who grow older with mental illness

\section{Ageism and psychiatric classification}

Up until the middle of the $20^{\text {th }}$ century, treatment for mental illness in later life was limited, as it was assumed that mental illness in older people was a part of the aging process (Hilton, 2005). When symptoms appeared for the first time in older age, it was common practice to search for neurological signs or evidence of decline related to organic disease, and 
other disorders were therefore neglected (Roth, 1955). Schizophrenia was conceptualized as a dementing illness and since dementia was known to be a progressing deteriorating disease course, it was seen as hopeless to aim for improved outcomes through research endeavors, even though Aloysius Alzheimer established around 1906 that dementia was a distinct illness with different characteristics.

In the United Kingdom, the term paraphrenia was used to distinguish schizophrenia in later life from that which was diagnosed earlier and lived with through older age. Paraphrenia, (experiencing hallucinations and delusions but without deterioration or disturbance of affective response), was thought to be quite different from late onset schizophrenia, (Howard et al., 2000) but the term was included in the ICD 9 (WHO, 1977) driven by the establishment of a distinct subspeciality of geriatric psychiatry in Europe (Howard et al., 2000), introduced in England in 1988 (Jolley, Kosky, \& Holloway, 2004). The terms late onset schizophrenia and paraphrenia have often been used interchangeably. Various challenges were made to beliefs about mental illness in later life when the relevance of functional illness for older adults was clarified. Bleuler (1943) examined 126 patients whose illness began after the age of 40 years and $4 \%$ had onset after age of 60 . Fifty per cent of late onset patients had symptoms indistinguishable from those seen in younger people with schizophrenia. In a study of 150 case records, half of the total number of cases were categorized as affective psychosis, $55 \%$ of which had occurred for the first time after the age of 60 (Roth \& Morrissey, 1952). Researchers concluded that affective psychosis, late paraphrenia, and acute confusion were distinct from senile and arteriosclerotic psychosis, the two main causes of progressive dementia in old age. Therefore their hypothesis was confirmed, that is, mental illness in older people wasn't all about dementia. Similarly, a follow up study investigating 464 case records found that 450 cases could easily be placed in one of 5 diagnostic groups; acute confusion, affective psychosis, late paraphrenia, 
arteriosclerotic psychosis, or other, and had distinct differences in outcome such as death or discharge home (Roth, 1955). It is possible that evidence such as this did not gain the attention of the professional community at the time as it represented the efforts of a few interested individuals confronting established and accepted truths. It also highlights how the process of getting evidence into practice can be an exceptionally long one, an issue that remains a challenge today.

\section{Historical development of psychiatric classification related to schizophrenia}

'Dementia praecox' was originally named by Kraeplin in 1893 and identified by Bleuler in 1911 as 'schizophrenia' (Snowden, 2008). It was thought to be a dementing illness in young people, but later evidence questioned this idea. Bleuler (1978) followed the natural course of schizophrenia in a large group of patients over their life course in a psychiatric hospital and found multiple outcomes; complete recovery, profound dementia, and complete disability. These early insights into the prognosis of schizophrenia contributed to its understanding but it was not until 1987 that it was acknowledged that schizophrenia could be diagnosed for people over the age of 40 , classed as late onset schizophrenia. "Very late onset" is a term sometimes used for patients with onset of schizophrenia-like psychosis after age 60 (Reeves \& Brusterm 2008).

Practitioners in the psychiatric field have two manuals that serve as primary resources: The International Classification of Diseases (ICD) published by the World Health Organization (WHO) and the Diagnostic and Statistical Manual of Mental Disorders (DSM) published by the American Psychiatric Association (APA). These two closely related manuals have been used for mental illness classification since 1948 and 1952 respectively.

The DSM was originally intended for use in the United States but through the years of its revisions has had considerable influence internationally. It is important to understand that it was developed in a particular cultural context that was based on research with western 
populations and thus caution should be used in automatically applying criteria to non-western populations (APA, 1987).

Psychiatric classification has developed considerably over the past 100 years, resulting in over 400 diagnoses that are now available. In the early stages of classification development, medical practitioners grappled with many issues. There seemed to be as many classification systems as there were people developing them (Collier, 2008), making it difficult to communicate a collective understanding of mental illness and preventing systematic collection of statistical information on mental illness. Even now with sophisticated standardized approaches to classification, there may be a tendency to support a particular classification system depending on a theoretical position (Snowden, 2008).

\section{Schizophrenia and older people}

Although older adults with schizophrenia rate health as their top priority, they have poorer physical health than peers of the same age without schizophrenia (Jones, Vahia, Cohe, Hindi, \& Nurhussein, 2009). Schizophrenia has disruptive effects on many aspects of life that may affect older adults. For example, women with schizophrenia are less likely to marry, bear children, and raise their own children than are women in the general population (Dickerson, 2007). Older adults with schizophrenia have increased mortality that may be due to a variety of factors, such as patient characteristics, attitudes of health care providers, and institutional factors (Jones, et al, 2009). Patient factors may include such things as unhealthy lifestyle, adherence to treatment, and financial resources. Health care professionals' attitudes and relationships with other health care providers are another factor. Institutional factors include cost of services, location, insurance standards, and information networking.

Medical classifications represent a specific set of predetermined defined symptoms and duration that are useful for informing pharmacological treatment protocols. They may not be as helpful, however, in providing meaningful understanding of a person's experiences, as 
there is a great deal of variability in how symptoms present in individuals across the lifespan (Collier, 2007). The heterogeneity of outcomes is consistently reported (Ban, 2004; Howard et al., 2000) and because of this variability, with different outcomes in different groups, it is important for future research to focus on individual experience (Collier, 2007).

Focusing on single characteristics, rather than one broad category, can lead to a more meaningful understanding of how symptoms of schizophrenia change during the aging process. For many years, the term "burned out" has been used to describe the changes that are observed in long term schizophrenia, sometimes implying that symptoms such as hallucinations disappear in the aging process (Palmer et al., 1999). A review of research related to the use of the term "burn out", however, indicates that there is no consistent definition of the term. It seemed to be used in practice to suggest that the patient was no longer acutely ill but may still be disabled by such symptoms as apathy and neglected hygiene (Collier, 2007). In addition, there is inconsistency in outcome studies regarding whether people get better or worse as they age. For those who develop schizophrenia for the first time in later life, it has been suggested that retirement, bereavement, disability, and financial difficulties may contribute to the precipitation of the symptoms (Howard et al., 2000).

In recent years a consensus report representing 'unprecedented transatlantic agreement' has been published by Howard et al (2000) and the international late onset group who argue that there is sufficient evidence to justify recognition of two illness classifications of schizophrenia in later life; late onset after age of 40 and very-late-onset schizophrenia like psychosis after the age of 60 . They recommend the use of standardised criteria but state that there should be no criterion that excludes a diagnosis of schizophrenia based on late age at onset and there is no separate codable diagnosis for late onset.

\section{Implications}


Nursing research on schizophrenia during the last 10 years has focused on recovery philosophies emphasizing individual strengths and the adaption of positive expectations. Much of this, however, continues the exclusion of people who are in older age groups. Questions remain about how older people, as they have aged, have learned to manage their problems (Collier, 2010). Therefore, challenges remain in tackling this age discrimination for older people with schizophrenia.

It is important to consider how nurses' beliefs about persons with long-term schizophrenia may affect practice. Nurses should reflect on their own attitudes as to how medical frameworks such as the psychiatric classification systems may have influenced their understanding of mental illness. They should also carefully consider how a negative view of schizophrenia in older adults may affect nursing interventions. If nurses have negative beliefs about the abilities and potential of older people with schizophrenia, they may refrain from implementing interventions that could be helpful (Collier, 2007). Traditional views regarding decline may prevent rehabilitation efforts in later life schizophrenia (Palmer et al., 1999).

We all have a responsibility to review our attitudes and the effect on our practices. Some studies suggest that negative physician attitudes are a major barrier to delivering adequate health care to people with schizophrenia, with psychiatrists and primary care physicians showing the same level of stigma towards such people as those in the general population (Jones, et al., 2009). Jones, et al. (2009), in a survey of 24 primary care providers and 27 psychiatrists in New York, found that both groups had slightly negative stereotypes and attitudes toward older adults with schizophrenia. Data from the study suggested, however, that the failure of this population to receive adequate care may not be from clinician's negative attitudes and behaviors, but from poor communication with other health care providers. 
A study by Hellzen, Kristiansen, and Norbergh (2003) explored Swedish nurses' attitudes toward older residents with long-term schizophrenia. They found that nurses with long experience were less sensitive in their relationship with the resident than less experienced nurses, suggesting that long work experience can have a negative effect on nurses' attitudes towards the resident. Findings from a study by Leutwyler and Wallhagen (2010) suggest that trust between health care providers, the health care system, and the older adult with schizophrenia is an important factor in physical health outcomes.

There is a need for more research concerning attitudes and behaviors of practitioners caring for older adults with schizophrenia. Older people with schizophrenia are still subject to discrimination from professionals on the basis of age and are generally less likely to be treated with modern medications that have many benefits, such as a reduced risk of tardive dyskenesia (Harvey, 2005). The reasons for this are suggested as;

- therapeutic nihilism: a lifetime of chronic symptoms and poor treatment response may lead clinicians to think that there is nothing that can be done

- ageist bias: it is not worth intervening if a person is older and therefore resources are not allocated

- financial bias: they may never have worked or paid taxes because of life long disability and so never contributed to society. There may therefore be reluctance to provide more expensive medications particularly in insurance led systems.

- absence of constituency - few advocates, concentrated on younger people. may not have relatives to help. (Harvey, 2005, p. 181).

We know little about the specific experiences of older adults living with schizophrenia. Nurses working in mental health settings need to be careful in assigning premature labels to older adults with mental illness. Rather than making assumptions about 
levels of disability, it is important for nurses to 'suspend disbelief' (Perkins \& Tice, 1995) and attempt to explore a person's strengths not yet discovered and be prepared to elicit the life history and personal stories of older people with schizophrenia.

\section{Conclusion}

There is a need for more research related to schizophrenia in older adults, not only to be better informed regarding the prognosis for individuals and best practices for treatment, but also to understand their unique experiences. It has been suggested that the term "burned out" should not be used in practice, as it can be misleading and possibly negate a proactive reassessment or intervention that could be helpful to the individual (Collier, 2007). There should be a clear boundary between late-onset schizophrenia and other delusional disorders in middle and old age because confusion in terminology is a serious impediment to comparative international research (Riecher-Rossler, Förstl, \& Mesle, 1995).

Due to a lack of evidence and understanding about schizophrenia in older adults, it is possible that negative assumptions are made. Nurses need to reflect on their practice with older adults with schizophrenia and attempt to create positive opportunities for interventions and personal development. 


\section{References}

American Psychiatric Association (APA) (1987). Diagnostic and Statistical Manual III. Washington, D. C: American Psychological Association.

Ban, T.A. (2004). Neuropsychopharmacology and the genetics of schizophrenia. A history of the diagnosis of schizophrenia. Progress in Neuro-Psychopharmacology and Biological Psychiatry, 28, 753-762.

Bleuler, M. (1943) Die Spatschizophrenen krankheitsbilder. Fortschritte der Neurologie und Psychiatrie 15:259-290 cited in Howard, R., Rabins, P.V., Seeman, M.V., \& Jeste, D.V., International late onset schizophrenia group. (2000). Late-onset schizophrenia and very-late-onset schizophrenia-like psychosis: An international consensus. American Journal of Psychiatry, 157, 172-178.

Bleuler, M. (1978). The schizophrenia disorders long term patient and family studies. New Haven, CT. Yale University Press. Cited in Harvey, P.D. (2005) Schizophrenia in late life. Ageing effects on symptoms and course of illness. Washington, DC: American Psychological Association.

Bridge, T.P., Cannon, E., \& Wyatt, R.J. (1978). Burned-out schizophrenia: Evidence for age effects on schizophrenic symptomology. Journal of Gerontology, 13(6), 835-839.

Cohen, C. I., Vahia, I., Reyes, P., Diwan, S., Bankole, A. O., Palekar, N., Kehn, M., \& Ramirez, P. (2008). Schizophrenia in later life. Clinical symptoms and social wellbeing. Psychiatric Services, 59(3), 232-234.

Collier, E. (2005). Latent age discrimination in mental health care. Mental Health Practice, $8(6): 42-49$.

Collier, E. (2007). Challenging the concept of "burned-out" schizophrenia; Implications for practice. Mental Health Nursing, 27(4), 14-18. 
Collier, E. (2008). Historical development of psychiatric classification and mental illness. British Journal of Nursing, 17(14), 890-894.

Collier, E. (2010) Confusion of recovery: One solution. International Journal of Mental Health Nursing, 19, 16-21.

Dickerson, F. B. (2007). Women, aging, and schizophrenia. Journal of Women and Aging, 19(1/2), 49-61.

Harvey, P.D. (2005). Schizophrenia in late life. Ageing effects on symptoms and course of illness. Washington, D.C.: American Psychological Association.

Hellzén, O., Kristiansen, L., \& Norbergh, K.G. (2003).Nurses’ attitudes toward older residents with long-term schizophrenia.Journal of Advanced Nursing, 43(6), 616-622.

Hilton, C. (2005) The clinical psychiatry of late life in Britain from 1950 to 1970: A overview. International Journal of Geriatric Psychiatry 20(5), 423-428.

Howard, R., Rabins, P.V., Seeman, M.V., \& Jeste, D.V. and the International Late-onset Schizophrenia Group (2000) Late-onset schizophrenia and very-late-onset schizophrenia-like psychosis: An international consensus. American Journal of Psychiatry, 157(2), 172-178.

Jolley, D., Kosky, N., Holloway, F. (2004). Older people with long standing mental illness; The graduates. Advances in Psychiatric Treatment, 10, 27-36.

Jones, S.M., Vahia, I.V., Cohen, C. I., Hindi, A., \& Nurhussein, M. (2009).A pilot study to assess attitudes, behaviors, and inter-office communication by psychiatrists and primary care providers in the care of older adults with schizophrenia. International Journal of Geriatric Psychiatry, 24, 254-260.

Leutwyler, H. C., \& Wallhagen, M. I. (2010).Understanding physical health of older adults with schizophrenia.Building and eroding trust.Journal of Gerontological Nursing, $36(5), 38-45$. 
Palmer, B.W., Heaton, S.C., \& Jeste, D.V. (1999). Older patients with schizophrenia: challenges in the coming decades. Psychiatric Services, 50(9), 1178-1183.

Perkins, K., \& Tice, C. (1995) A strengths perspective in practice: Older people and mental health challenges. Journal of Gerontological Social Work, 23(3/4), 83-97.

Reeves, R., R, \& Brister, J. C. (2008). Psychosis in late life. Emerging issues.Journal of Psychosocial Nursing, 46(11), 45-52.

Riecher-Rössler, A., Rössler, W., Förstl, H., Mesle, U. (1995). Late-onset schizophrenia and late paraphrenia. Schizophrenia Bulletin, 21(3): 345-354.

Roth, M. (1955). The national history of mental disorder in old age. Journal of Mental Science, 101, 281-301.

Roth, M., \& Morrissey, J.D. (1952). Problems in the diagnosis and classification of mental disorders in old age. Journal of Mental Science, 98(410), 66-80.

Snowden, A. (2008). Schizophrenia: Early history of diagnosis. Nurse Prescribing, 6(10), 444-445.

Webb, A. K., Jacobs-Lawson, J. M., \& Waddell, E. L. (2009). Older adults' perceptions of mentally ill older adults. Aging and Mental Health, 13(6), 838-846.

World Health Organization (WHO) (1977). International classification of diseases $9^{\text {th }}$ edition. WHO, Geneva. 